\title{
Heimatverbunden und weltoffen
}

\author{
Wege und Möglichkeiten der Bildung und Erziehung im Geographieunterricht ${ }^{1}$
}

Kurt Bösiger

Heimatverbunden und weltoffen bedeutet nicht Widerspruch, sondern gesundes Wechselverhältnis - die beiden Begriffe schließen sich also nicht aus, sondern ergänzen sich wechselseitig (Lit. 8). Als prägnante Formel drücken sie zwei wesentliche Bildungsziele der modernen Schulgeographie aus, denn in der Spannweite zwischen den Stoffbereichen Heimat und Welt liegen die vielgestaltigen Aufgaben unseres schönen Faches. Das Wort $\mathrm{Ver}$ bundenheit klingt wärmer, gefühlsvoller - Offenheit tönt sachlicher, bedeutet waches Interesse und Aufnahmebereitschaft des Geistes. Heimat ergreift - noch heute - das Gemüt, während die übrige Welt in erster Linie mit dem Verstand erfaßt wird; eine scharfe und allgemein gültige Grenzziehung ist natürlich nicht möglich.

Beginnen wir - einem alten methodischen Prinzip folgend - mit der räumlichen und geistigen Nähe, bevor wir unsere Gedanken in die Ferne schweifen lassen! In seinem höchst anregenden Werk «Die Schweizer sind anders» leitet der bekannte Publizist Adolf Guggenbühl ein Kapitel zum staatsbürgerlichen Unterricht kurz und bündig mit dem Satz ein:

"Die stärkere Betonung des Heimatlichen auf allen Gebieten ist die wichtigste Grundlage der Erziehung zur Demokratie» (Lit. 6, S. 287).

Er fordert sogar die Festlegung dessen, was zum eisernen Bestand des Wissens und Könnens gehört und gibt seinem Erstaunen darüber Ausdruck, daß viele junge Lehrer für eine Ferienreise bedeutend größere geistige Vorbereitungen treffen als in bezug auf den Wirkungsort, an dem sie jahrelang tätig sind (Lit. 6, S. 281 ff.). Guggenbühl ist überzeugt, daß der Durchschnittsschweizer von seinem Staat trotz allen Schulreformen herzlich wenig weiß und verlangt nicht etwa nur die Förderung der Heimatkunde als Fach, sondern postuliert die unmittelbare Umgebung als Ausgangspunkt für andere Fächer. Er weiß aber auch, wie "altmodisch» seine Forderungen sind und zitiert die nachstehenden Sätze:

"Was nutzet uns, wenn wir hersagen können, wieviel Gold Peru, wieviel Silber Potosí und Spanien einträgt, wie zahlreich die französischen Kriegsheere, wieviel Herzogtümer in Deutschland, wie weitschichtig Moskau, wie stark die Ottomanen, wie volksreich China, wie streitbar Persien, mit einem Wort, wie die ganze Welt beschaffen, wenn hingegen uns unbekannt bleibt, in welchen Dingen die wahre Kraft und Macht der Eidgenossenschaft bestehet, wenn wir Fremdlinge in unserem Vaterlande, dessen Geschichte und Staatskunde sind?»

Diese Worte stammen aus einer Publikation von Franz Urs von Balthasar:

«Patriotische Träume eines Eidgenossen, von einem Mittel, die veraltete Eidgenossenschaft wieder zu verjüngen.»

Das Büchlein erschien 1758 in Basel, doch war wegen der Zensur der Erscheinungsort wie folgt angegeben: «Freistadt, bei Wilhelm Tells Erben.»

Nach dieser kleinen Dokumentation zur dauernden Aktualität heimatkundlicher Bemühungen, wollen wir uns der Begriffsbestimmung aus geographischer Sicht zuwenden. Heimatkunde im engeren Sinne umfaßt die elementare Kenntnis des Heimatortes und seiner Umgebung, im weiteren Sinne geographische Vaterlandskunde.

"Es gehört zum Menschen schlechthin, daß er in dieser Welt irgendwo, daheim' sein muß. Unsere menschliche Existenz erschöpft sich nicht im bloßen Vorhandensein und Irgendwo-Wirken. Sie bedarf eines irdischen Ortes und Raumes, auf den sie als Ganzheit unmittelbar bezogen ist und von dem her sie geprägt und gehalten wird. Pestalozzi hat von ,Individuallage', Spranger von ,Eigenwelt' gesprochen» (Lit. 1, S. 50).

Spranger ging noch weiter und äußerte sich schon 1923 in seinem berühmten Vortrag über den "Bildungswert der Heimatkunde» (Lit. 9) dahin, daß im Heimaterlebnis etwas tief Religiöses mitschwinge, und zwar auch bei dem, der es nicht eingestehen wolle. Er definiert Heimatkunde als "geistiges Wurzelgefühl». Demnach erfassen wir in ihr die natürlichen und geistigen Wurzeln unserer Existenz, und auf dieser Tatsache beruht ihr hoher Bildungswert. Sie strebt also - wie jede echte Bildung - nach der Erfassung des Menschen in seiner geistigen Struktur.

Heimatkunde darf - bei aller Bedeutung von Ge- 
schichte, Sage, Tradition und Brauchtum - nicht im Historischen steckenbleiben. Neben dem Gestern muß das Heute, ja sogar das Morgen, einbezogen werden. Die Verknüpfung von Vergangenheit und Gegenwart in der heimatlichen Sphäre bildet das Fundament zum Verständnis fremder Landschaften; denn der Schüler muß auch die Heimatbezirke anderer Menschen kennen und achten lernen. Die Heimatkunde schafft die Begriffe für die Länderkunde und vermittelt die Grundanschauungen aller geographischer Erkenntnisse. Deshalb muß sie alle Klassen durchziehen, kommt doch dem Vergleich mit den gewohnten Verhältnissen und Maßstäben während der ganzen Schulzeit große Bedeutung zu.

Ein Beispiel zur Illustration: Gewarnt durch die verhängnisvollen Folgen einer übersteigerten Technisierung, hat man in den letzten Jahren die Bedeutung von Natur- und Heimatschutz vermehrt erkannt. Die bloße Feststellung von anthropogenen Landschaftsschäden und ihren Folgen - etwa Entwaldung, Bodenzerstörung, Gewässerverschmutzung oder unorganische Verstädterung - vermag das Verantwortungsbewußtsein der Schüler gegenüber der Heimatlandschaft zu wecken, und aus dieser Einsicht erwachsen am ehesten die Forderungen nach Landschaftsschutz und Planung. Am aktuellen Fall der näheren Umgebung können die Probleme der Landflucht und Verstädterung eindrücklich diskutiert und damit die sachliche Kritikfähigkeit des jungen Staatsbürgers gefördert werden. Durch die 1961 erfolgte Annahme des neuen Natur- und Heimatschutzartikels 24sexies in die Bundesverfassung hat das Schweizervolk anerkannt, daß Naturschutz Lebensschutz bedeutet. Wir sind wohl der einzige Staat der Welt mit einem solchen Verfassungsartikel. Eng mit dem Heimat- und Naturschutz verknüpft sind Fragen der Landes-, Regional- und Ortsplanung, wozu die Geographie gleichfalls wichtige Grundlagen liefert. Rechtzeitige Planung im schweizerischen Bereich der Basler Region hätte beispielsweise unser Birswasser und die Luft unserer besten Wohnlagen vor Schändung bewahrt und für einen harmonischen Ausbau unserer heimatlichen Kulturlandschaft gesorgt.

Wir haben an diesen Beispielen erkannt, daß die Heimatkunde in stufengerechter Weise die gemeinsame Erfahrungsgrundlage für unsere Schüler schaffen muß, wobei sie zudem ausgezeichnete Möglichkeiten zur Überwindung der Fächertrennung und zu lebensnaher Unterrichtsgestaltung bietet. In unserem Zeitalter intensiver Verstädterung muß allerdings der Begriff "Heimat» neue Akzente erhalten. $\mathrm{Ob}$ die einheimische Bevölkerung noch überwiegt oder ob die Zugewanderten vorherrschen
- der Lebensraum der Stadt wird zur Heimat. Setzen wir jedoch «Heimat» in Anführungszeichen. Denn zweifellos bewirkt die Verstädterung eine Lockerung des ursprünglichen Heimatbewußtseins; die mannigfachen Probleme der fluktuierenden Bevölkerung liegen häufig in der mangelnden Verwurzelung im neuen Wohn- und Arbeitsbereich begründet. Leider scheint bei uns die Stadtgeographie immer noch ein Stiefkind geographischer Betrachtung zu sein. Den Mangel an schulmethodisch ausgewerteten und Interessenten zugänglich gemachten Beispielen dieser Art in der Schweiz zu mildern, bezweckt unsere kleine Publikation für die Oberstufe: "Basel und seine Region in der Schulgeographie» (Lit. 2). Es scheint uns an der Zeit, daß auch die Schulgeographen den erzieherisch so wertvollen Gedanken einer nicht politisch, sondern kulturell und wirtschaftlich konzipierten Region Basel weitertragen helfen, nachdem die schon vor Jahren von Basler Geographen lancierte Idee einer planmäßigen Aktivierung und Erforschung der "Regio Basiliensis» nun auch in Kreisen von Wirtschaft und Verwaltung eingeschlagen hat. Es ist überraschend, wie einhellig positiv die Reaktion der Schüler über Einzel- und Gruppenarbeiten - verbunden mit Exkursionen - zur Agglomeration Basel wie auch zu Themen im weiteren Bereich unserer Heimat ausfällt. Voraussetzung solcher Arbeiten ist allerdings eine Einführung in die Technik der Einzel- und Gruppenarbeit.

Wiederum ein Beispiel: Das Arbeits-Studienlager meiner dritten Diplomklase in Sedrun GR ist eines der vielen, welche an den Basler Schulen im Laufe der letzten Jahrzehnte organisiert wurden. Die systematischen Vorbereitungen auf diese Schulkolonie setzten schon $1 \frac{1}{4}$ Jahre vorher ein, besonders aber im letzten halben Jahr. Unser Hauptanliegen bestand in der Förderung der selbsttätigen Schülerarbeit schon lange vor dem Lager. Ohne Anleitung können unsere Schüler selten wirklich selbständig arbeiten, vor allem nicht direkt aus der Naturanschauung heraus: Die Fähigkeit zum Erkennen der Probleme muß geübt werden. Gründliche Literaturstudien vor der Kolonie und in der Kolonie, ergänzt durch die Anschauung im Gelände - beide Phasen verbunden mit Einführungen unter der Anleitung des Lehrers - scheinen uns die fruchtbarste Kombination für obere Klassen zu sein. Die angewandte Methode des Gruppenunterrichts ermöglichte auf natürliche Weise eine Einführung in das wissenschaftliche Arbeiten, wobei die Schüler - mehr oder weniger selbständig — vertiefte Kenntnisse über ein neues Stoffgebiet erwarben (Lit. 3). Wieviel Ausdauer und Energie von Schüler- und Lehrerseite notwendig sind, mag die Tatsache illustrieren, daß je Studie und Schüler 2 bis 
4 Entwürfe, 1 bis 2 Reinschriften und mehrfaches Übertragen auf Matrizen nötig waren und alles vom Lehrer korrigiert werden mußte. Im Hinblick auf den offenkundigen Arbeitseifer haben wir bei etwas einseitiger Spezialisierung der Schüler gelegentlich ein Auge zugedrückt, etwa wenn die innert 24 Stunden von der jeweiligen Küchenmannschaft in französischer Sprache verfaßten Tagesberichte $z u$ deutlich den Stilstempel ihres zweisprachigen Klassenkameraden trugen oder wenn die guten Matrizenschreiber zu sehr nur schrieben, die begeisterten Zeichner zu viel Titelblätter entwarfen.

Gut organisiert und richtig durchgeführt, bildet die Schulkolonie zweifellos eine optimale Form des Arbeitsunterrichts. Fernab von der gelegentlich drückenden Enge der Schulstube, nach Möglichkeit in der freien Natur, kann die Arbeit oft intensiver und vom Schüler her gesehen auch initiativer betrieben werden. Nehmen wir beispielsweise das Leitmotiv "Natur und Volk» und stecken uns das Ziel, die Gemeinschaftsidee zu fördern, indem wir das Leben der bäuerlichen Familie und im weiteren Sinne das Leben einer Dorfgemeinschaft zum Gegenstand unseres Studiums wählen!

Die synthetische Betrachtungsweise der Geographie verhindert, daß über dem Vielerlei des Neuen das Ganze vergessen wird; denn der naturnahe Geographieunterricht in der Schulkolonie zeigt die Wechselbeziehungen zwischen Boden, Klima, Vegetation und Mensch auf. Die Untersuchung der einzelnen Gegenstände bietet zugleich den lebenserfüllten Stoff für die andern Fächer. So können - in natürlicher Querverbindung - in der Geschichte die genossenschaftlichen Einrichtungen der Dorfbewohner studiert werden, was das Verständnis für den staatsrechtlichen Aufbau unserer Demokratie weckt. Der Deutschlehrer findet in der Form von Exkursionsberichten und Beschreibungen gute Aufsatzthemen, die Naturkunde kann mithelfen, Gedanken des Landschaftsschutzes in den Mittelpunkt des Gesamtthemas zu rücken, und die Mathematik verzichtet für einmal auf abstrakte Gedankengänge und widmet sich der gegenständlichen Aufgabe einer Feldmessung. Wie der fremdsprachliche Unterricht dem Kernfach Geographie dienlich sein kann, haben wir oben angedeutet, und auch das Zeichnen kommt gewiß nicht zu kurz. So ergibt sich eine detaillierte Sicht der Objekte im Raum, verbunden mit einer einheitlichen Gesamtschau eines kleinen Landschaftsausschnittes. Diese Verbindung ist für die Schule besonders wertvoll. Es ist also nicht Fachegoismus, sondern die Eignung der Geographie, die unsere Vorschläge zur Gestaltung der Schulkolonie bestimmen.

Aus den angeführten Beispielen zur Agglomeration Basel und über Studienlager in einer ländlichen $\mathrm{Ge}$ - meinde unseres Landes lassen sich einige Rückschlüsse methodischer Art ziehen. Wir sind der Überzeugung, daß der Geographielehrer die Methode des Schilderns und Dozierens maßvoll anwenden sollte, auch auf der Mittelstufe. Geographisch gut schildern kann in der Regel nur, wer selbst etwas gesehen und erlebt hat. Dabei vergessen wir keineswegs, daß geographisches Darstellen heute noch in meisterhaften beschreibenden oder erklärenden Schilderungen gipfelt und durchaus in pakkender Erzählform geboten werden kann. Wieviel Lehrer verfügen aber über diese künstlerische Gabe - gerade unter den in unserer hektischen Zeit so wertvollen sachlich-nüchternen Typen naturwissenschaftlicher Richtung? Auch die entwickelnd-darstellende Methode hat in der Geographie nach wie vor ihre Berechtigung, ist aber gleichfalls mit $\mathrm{Ma} \beta$ anzuwenden und durch arbeitsunterrichtliche Verfahren vermehrt aufzulockern - wir meinen in diesem Fall im Prinzip alle Stufen. So verstanden, wird der exemplarische Unterricht - zumindest an der Oberstufe - zu einer dominierenden Methode: denn nicht die Anzahl der behandelten Einzelbeispiele ist letztlich entscheidend, sondern die Tiefe der fachlichen Durchdringung. Selbstverständlich wird die Behandlung ausgewählter Landschaften in Sinne der Schwerpunktbildung erst dann ersprießlich, wenn gesicherte topographische Grundkenntnisse vorhanden sind und der Gesamtzusammenhang in der Form von Überblicken gewährleistet ist. Wir glauben, am Exempel der Schulkolonie gezeigt zu haben, daß ein guter Geographieunterricht bewußt über die Grenzen des Faches hinausführt, in seiner Mittlerrolle zwischen Natur- und Geisteswissenschaften die Einheit der Bildung sichtbar werden läßt und so der Konzentration dient. Damit aber wird die Geographie zur echten Wirklichkeitskunde!

Es sei nicht bestritten, daß dem Lehrer aus der Eigenart und der Stoffülle des Faches zusätzliche Probleme erwachsen. So werden etwa in den Sprachfächern wesentliche Teile des Stoffes durch die Anwendung in Wort und Schrift ständig und automatisch wiederholt. In der Geographie geraten behandelte Gebiete - ähnlich wie in der Geschichte leichter in Vergessenheit. Um so wichtiger ist es für den Lehrer, zu überlegen, welche Kenntnisse und Einsichten er als grundlegend betrachtet. $\mathrm{Da}$ das Hauptgewicht nicht der sogenannten «Briefträgergeographie" gebührt, dürfte sich ohne Schaden noch mehr herumsprechen! Da mit der erstmaligen Behandlung kein dauerndes Behalten des Lehrstoffes erreicht werden kann, sind Wiederholungen beim besten Unterricht unerläßlich, besonders in den Realienfächern. Durch Versuche hat man festgestellt, daß nach zwei Tagen durchschnittlich zwei Drittel des Gelernten vergessen sind. Daher kommt der ersten, bald durchzuführenden Repetition ent- 
scheidende Bedeutung zu; die folgenden Wiederholungen dienen nur noch der Festigung. Sie müssen den Examenscharakter verlieren und durch vorbereitete Fragen zu spannenden und mit Freude erwarteten Lektionsteilen gestaltet werden. Es soll noch heute Lehrer geben, die den Wert der Repetitionen verkennen und deshalb dafür in den Schulstunden zu wenig Zeit einräumen - hoffentlich sind keine Geographen darunter!

Heimatverbunden und weltoffen lauten die Titelworte, und Sie finden vielleicht, die räumliche Nähe habe eine Vorzugsbehandlung erfahren. Ist es nicht so - könnten Sie einwenden - daß in einer Zeit der Hochkonjunktur immer mehr Schüler ihre Ferien im Ausland verbringen und sich das Schwergewicht geographischen Erlebens und Erkennens einfach auf weiter entfernte Autoziele verlagert? Die Erfahrung lehrt leider das Gegenteil: Je mehr Kilometer durchrast werden, um so oberflächlicher sind meistens die Kenntnisse. Fragt man auf der Oberstufe überraschend nach Grundlagenwissen, so ist der Ertrag bescheiden. Andererseits lernen immer weniger Jugendliche ihre Heimat auf Schusters Rappen kennen, und manchmal fehlt für Wanderungen sogar die geeignete Ausrüstung, namentlich in Mädchenklassen. Die Schlußfolgerung scheint klar: Die Geographie der Schweiz muß nach wie vor auf allen Schulstufen das gebührende Schwergewicht erhalten. Mit andern Worten: Die Weltoffenheit soll auf der Basis soliden heimatlichen Grundlagenwissens, das als Erfahrungsmaßstab die Vergleichsgröße darstellt, angestrebt werden; wir postulieren demnach eine Weitung des Horizontes von innen heraus. Nur so vermögen unsere Schüler das oft allzu verlockende fremde Gedankengut sachlich zu erfassen und vernünftig einzuordnen. Auch dazu ein Beispiel: Unsere Binnenlage, die Rohstoffarmut und die große Bevölkerungsdichte ketten uns zwar ans Ausland. Die damit verbundenen komplexen Wirtschaftsbeziehungen der Schweiz kann aber nur verstehen, wer auch die Verhältnisse innerhalb unseres Landes wirklich kennt. Der Schweizer Bürger soll wissen, woher die wichtigsten Gebrauchsartikel bezogen werden und warum dies so ist. "Das gehört in der gegenwärtigen Zeit so gut zur allgemeinen Bildung wie die Kenntnis der griechischen und römischen Götterwelt» - schrieb schon 1916 Prof. Dr. E. Letsch (Lit. 7).

Damit stecken wir mitten in der Thematik um die Bedeutung der Geographie für die staatsbürgerliche Bildung und Erziehung. Selbstverständlich ist die Geographie als solche nicht staatsbürgerlicher Unterricht, sondern sie liefert dank ihren Möglichkeiten zu fruchtbaren Querverbindungen lediglich Beiträge dazu. Insofern ist letztes Ziel des Geographieunterrichts die Persönlichkeitsbildung.
Seit Jahren pflege ich an der Oberstufe die Diskussion aktueller Themen mit geographischem Gehalt. Nach einleitenden Schülerreferaten oder einer kurzen eigenen Einführung ins Problem provoziere ich jeweils möglichst viele Äußerungen. Oft erreichen solche Aussprachen ein erfreuliches Niveau. Was jedoch in zunehmendem Maße unangenehm berührt, ist der Umstand, daß es - neben kulturbewußten Votanten eine überraschend große Minderheit von bedingungslosen Anbetern des technischen Fortschritts gibt, denen die Erhaltung traditioneller kultureller Werte wenig oder nichts gilt und die jede Frage nach einem rein materialistischen Nützlichkeitsstandpunkt beurteilen. Am extremsten kommen diese Meinungen oft bei der Diskussion städtebaulicher Fragen zum Ausdruck, möchten doch einige Schüler die Altstadt Basels ganz oder doch mit Ausnahme des Münsterhügels durch Bauten aus Glas und Beton ersetzen! Die Schönheit unersetzlicher ehrwürdiger Bauten steht offenbar bei einem Teil unserer Jugend nicht mehr hoch im Kurs, sobald der Moloch "moderne Nützlichkeit» mit neuen Abbruchforderungen auftritt. So wird etwa die Einrichtung des Kantonalen Lehrerseminars in einem nach altem Stil renovierten Gebäude von Schülern $11 \frac{2}{2}$ Jahre vor der Maturitätsprüfung als verschwenderisch und unsinnig bezeichnet; Renovationen von alten Fassaden bei modernem Innenausbau gelten als ,unehrlich', Landschaftsund Naturschutz werden höchstens dann geduldet, wenn die alles bewegende Wirtschaft keine oder nur unbedeutende Opfer bringen muß.

Gewiß haben wir hier einseitig nur einige extreme Äußerungen herausgegriffen, doch wirft das unzweifelhafte Schwinden des Traditions- und Kulturbewußtseins Fragen von größter Tragweite auf: Wieweit sind unsere Schüler trotz der ihnen vermittelten allgemeinen Bildung ernstlich von der Vermaterialisierung und Verflachung des Lebens bedroht? Handelt es sich um ungereifte Jugendmeinungen oder werden solche Auffassungen bald zur herrschenden Tendenz? Was geschieht, wenn sich die Grenze zwischen dem durch die Hochkonjunktur geförderten Materialismus zum politisch untermauerten Materialismus des Ostens immer mehr verwischt? Wie lange dauert es dann, bis eine gröBere Minderheit unsere Landesverteidigung als illusorisch, zu teuer und zu wenig nützlich in Frage stellt? Für welche innern Werte will sich unsere verwöhnte junge Generation überhaupt noch opferfreudig einsetzen? $\mathrm{Da} ß$ es bei solchen und ähnlichen Fragen um Existenzprobleme unseres Staates und der freiheitlichen Welt geht, dürfte auf der Hand liegen. Antworten zu finden, ist nicht nur Sache der zuständigen Schulbehörden, sondern die Aufgabe 
jedes Lehrers. Der Fachvertreter allein kann sie nicht lösen - er benötigt die Hilfe der Hauptfachpädagogen. Sicher scheint uns dies: Wenn im totalitären Osten das gesamte Erziehungswesen vom Kindergarten bis zur Universität im Dienste der kommunistischen Ideologie steht, genügen bei uns bescheidene Teillösungen auf dem Gebiet der staatsbürgerlichen Bildung nicht mehr. Es ist bequem, aber zu billig, allzu vertrauensselig auf die innere Kraft der Demokratie zu bauen. Schenken wir jeder auf seine Weise - diesen Problemen inskünftig wieder mehr Aufmerksamkeit! Aber seien wir uns klar darüber, daß wir keine Dressur zum gehorsamen und manipulierbaren Staatsbürger wünschen. Letztes Ziel der staatsbürgerlichen Bildung sei für alle Fächer die Erziehung zur mitbürgerlichen Verantwortung, also die Stärkung des Gemeinschaftsgefühls. Erziehung zur Gemeinschaft erfolgt durch das Gemeinschaftsleben, d. h. im Elternhaus, in der Schule, in der Schulkolonie sowie im Rahmen der Gemeinde; sie ist demnach nur bedingt lehrbar. Die Schule kann sicher wertvolle Beiträge leisten und auch die Grundkenntnisse der Rechtsformen der staatlichen und sozialen Organisationen vermitteln - die Alleinverantwortung für die staatsbürgerliche Erziehung wird sie aber in einem demokratischen Staat nicht übernehmen können.

Es schien mir in diesem Kreise wichtiger zu sein, einige Gedanken über das eigene Haus zu äußern, allerdings mit offenem Blick aus Fenster und Türen. Befürchten Sie also nicht, daß ich nun noch die Schleusen meiner Weltoffenheit für eine endlose Ferne öffne! Dies ist glücklicherweise gar nicht notwendig. Wenn Sie mir bis hierher gefolgt sind, haben Sie bemerkt, daß echte schweizerische Heimatverbundenheit das Interesse für die geistige und räumliche Ferne mit einschließt. Heimat und Welt oder vertraute Nähe und unermeßliche Weite gehören zusammen: sie sind durch Natur und Kultur aufs engste miteinander verflochten. Die Schweiz gehört zu Europa - und Europa zur übrigen Welt! Begnügen wir uns also damit, noch ein Fenster unseres Heimathauses exemplarisch zu öffnen, um einen Blick auf Europas Stellung zwischen den Weltmächten und den Entwicklungsländern zu werfen.

Als Folge seiner Selbstzerfleischung in zwei Weltkriegen hat Europa seine jahrhundertealte Vormachtstellung eingebüßt. Erste Großmacht der Erde wurden die USA, wichtigster Konkurrent die UdSSR, welche als Haupterbe des Zweiten Weltkrieges ihren Einflußbereich bis weit nach Mitteleuropa hinein ausdehnte und ihren Ring von Satellitenstaaten nach außen durch den ,Eisernen Vorhang' bald mehr, bald weniger dicht abriegelte. Das frei gebliebene europäische Restdrittel kann unter diesen Umständen seine Rolle als ,dritte Kraft' zwischen den vorläufig einzigen Weltmächten nur dann spielen, wenn sich die Einzelländer in geeigneter Form zusammenschließen. Über die Notwendigkeit einer Integration scheinen sich die meisten Völker und Staatsmänner des freien Westeuropa einig zu sein strittig hingegen bleiben die einzuschlagenden Wege und das Verhältnis von zentralistischer und föderalistischer Staatsstruktur. Das abendländische Europa bildet zwar dank den gemeinsamen drei Wurzeln - griechisch-römische Kultur der Antike, Christentum und Germanentum - in geistig-kultureller Hinsicht noch heute eine gewisse Einheit, und auch infolge seiner eigenständigen, wenn auch heterogenen historischen Entwicklung darf es als eigener Erdteil bezeichnet werden. Soviel als Anleihe aus dem Geschichtsunterricht - nun aber zur Geographie zurück! Rein geographisch betrachtet, streben die einzelnen Räume auseinander, denn Europa ist in horizontaler und vertikaler Richtung der am stärksten gegliederte Kontinent. Man könnte sagen, das Gemeinsame der europäischen Landschaften liege vor allem in ihrer physischen Vielgestaltigkeit, womit erhärtet wäre, daß die geographischen Grundlagen auf den erwähnten Zusammenschluß eher hemmend als fördernd einwirkten. Unbestreitbar bringt die einzigartige Kleinkammerung Europas eine gewisse Isolierung der abgelegenen Landschaftsräume mit sich. Im Unterschied etwa zum großreliefierten Asien oder zum auseinanderstrebenden Südamerika stehen jedoch die Teillandschaften Europas durch offene Pforten und Pässe miteinander in Verbindung, beispielsweise durch den Rhein oder den Gotthard. Die Gunst der Mittellage Europas auf der Landhalbkugel ermöglichte die Eroberung und Europäisierung fremder Kontinente. Im 20. Jahrhundert erleben wir die Gegenbewegung: die Zentrallage birgt die Gefahr in sich, daß ein geschwächtes Europa zwischen den Blöcken der Großmächte zermalmt wird. Noch vor kurzem war die Welt der Kampfplatz für die europäischen Interessen - heute ist das kleine Europa zur Nebenbühne des Weltgeschehens degradiert. Die Staatsmänner und Völker Europas vermögen sich nicht aus den geistigen Fesseln eines überholten Nationalismus zu befreien, um in der Form eines geeinten Blokkes die alte Gunst der räumlichen Situation wieder aufzuwerten. Geschichtliche Erfahrung und geographische Lage führen uns den erfolgversprechenden Weg klar vor Augen. Emil Egli formuliert diese Gedanken in prägnanter Form (Lit. 4, S. 47): "Die Gegenwart verlangt bewußt Wendung und Einsatz zu europäischem Denken. Der Zusammenbruch der politischen Weltkorona Europas und die moderne Verkehrs- und Nachrichtentechnik machen uns die Kleinheit unseres 
Erdteils wahrheitsgemäß bewußt. Das Zusammenrücken im heimatlichen kontinentalen Raum sollte uns leichter fallen und dringlicher erscheinen als je. Die Einzelstimme im europäischen Chor hat vor der Welt an Kraft verloren. Europa als Ganzem kommt im großen Weltspiel eine Hauptrolle zu.»

Damit ist das Tor zur weiten Welt erneut aufgestoßen. Ob es uns behagt oder nicht - schweizerisches und europäisches Denken allein genügen heute nicht mehr: Dem globalen Raum muß gleichfalls die gebührende Beachtung geschenkt werden. Oder können wir ernstlich die folgenden Tatsachen ignorieren?

Nur ein Fünftel der Weltbevölkerung lebt in sogenannten "entwickelten Ländern»; diese bescheidene Minderheit verbraucht jedoch rund ein Drittel des Welteinkommens und nimmt je Kopf und Tag im Durchschnitt mehr als 3000 Kalorien zu sich. Über die Hälfte der Menschheit muß sich mit durchschnittlich 1000 Kalorien weniger begnügen, d. h. sie hungert. $\mathrm{Zu}$ den rund $10 \%$ der Erdbevölkerung, die weit über der Mangelgrenze von 2500 Kalorien, also im Úberfluß leben, gehört - neben den Bevorzugten in westeuropäischen Ländern - u. a. auch das Schweizervolk. Untersuchungen haben gezeigt, $\mathrm{da}$ große Teile unserer Bevölkerung die weltweite Bedeutung echter Entwicklungshilfe noch nicht erkannt haben und ihre Ablehnung einseitig mit mißglückten Experimenten begründen. Offenbar muß wieder einmal die Schule sachliche Aufklärungsarbeit leisten! Zweifellos ist es neben der Geschichte der Geographieunterricht, der die Grundkenntnisse über fremde Länder und Völker vermittelt. Bei der Behandlung Afrikas, Asiens oder Südamerikas ergeben sich natürliche Anknüpfungspunkte, um dem reiferen Schüler die Notwendigkeit, aber auch Problematik der Entwicklungshilfe zu demonstrieren. Denn ohne das Verständnis und die bereitwillige Unterstützung breiter Volksschichten ist unsere Mitwirkung bei der Lösung des Weltproblems "Hunger» auf die Dauer kaum denkbar. Mit diesem Thema ist der Gedankenkreis «Heimatverbunden und weltoffen" geschlossen und zugleich dokumentiert, daß es sich nicht um ein Gegensatzpaar handelt, sondern um pädagogische Anliegen, die sich aufs schönste ergänzen. Um den Schülern das Problem der Entwicklungshilfe nahezubringen, können wir beispielsweise den folgenden Gedankengang zugrunde legen:

Bei aller Notwendigkeit finanzieller Unterstützung ist die Bildungshilfe in den ersten Rang zu stellen. Warum? Besser ausgebildete Menschen können sich, auf die Dauer gesehen, selbst helfen, während bloße Wirtschaftshilfe sich wie ein Tropfen auf einem heißen Stein verflüchtigen kann. Von Fall zu Fall ist genau zu prüfen, in welcher Weise die Ent- wicklung eines wirtschaftlich rückständigen Gebietes am zweckmäßigsten erfolgt. Einige Möglichkeiten: Geld, Waren, Werkzeuge, Werkstätten, Fabrikanlagen und vor allem Bildungshilfe durch die Entsendung von Experten und die Einrichtung von Musterbetrieben aller Art.

Warum ist das Problem der Entwicklungsländer in den letzten Jahren plötzlich so aktuell geworden? Die Antwort gibt uns die jüngere Geschichte: Wirtschaftlich mehr oder weniger entwickelte Länder hat es immer gegeben, und Entwicklungshilfe in bescheidener Form existierte schon längst, beispielsweise durch die christlichen Missionen. Infolge der stürmischen Entkolonialisierungswelle nach dem Zweiten Weltkrieg sind aber die unabhängig gewordenen Entwicklungsländer Afrikas und Asiens als dritte, politisch oft noch nicht fest verpflichtete Kraft zwischen der freien und der kommunistischen Welt in ungeahnter Weise in den Blickpunkt des Weltinteresses gerückt. Der mehr oder weniger kalte Krieg zwischen Freiheit und Diktatur wird vor allem um die Seele dieser jungen Staaten ausgetragen. Während die abendländischen Demokratien meist das Produkt eines langen geschichtlichen Prozesses sind, wollen die ehemaligen Kolonialvölker ihren Rückstand auf politischem und wirtschaftlichem Gebiet in kürzester Zeit aufho!en, was als kaum realisierbar erscheint. Aus Unkenntnis der natürlichen Voraussetzungen und der funktionalen $\mathrm{Zu}$ sammenhänge kommt es dann zu Fehlleistungen, die durch unzweckmäßige Entwicklungshilfe ungewollt gesteigert werden. Schlußfolgerung: Die Hilfe soll ïberlegt, zielbewußt und konzentriert sein. Deshalb setzt sie gründliche Studien über die Entwicklungsgrundlagen voraus - und hier liegt der Ansatzpunkt für den Geographen: Erste Voraussetzung für jede wirksame Unterstützung ist die Kenntnis der natur- und kulturräumlichen Verhältnisse des betreffenden Gebietes sowie die Vertrautheit mit der Psychologie der Landesbevölkerung. Es geht demnach darum, einen Einblick in die differenzierte Struktur der verschiedenen hilfsbedürftigen Räume zu gewinnen, und im Rahmen dieser Grundlagenforschung ist die Mithilfe der Geographie unentbehrlich. In der Praxis hat sich gezeigt, daß zuviele Entwicklungsländer die Lösung ihrer Probleme in einer kurzfristigen Industrialisierung oder in der Entwicklung des Fremdenverkehrs sehen. Oft müssen sie erkenen, daß der Kampf gegen den Hunger nur durch eine weniger spektakuläre Verbesserung der landwirtschaftlichen Produktion gewonnen werden kann.

Als nachahmenswertes Beispiel mag der Aufbau der schweizerischen Entwicklungshilfe in Nepal dienen, und als gutgelungenes Experiment sei die 1961 von der "Basler Stiftung zur Förderung von Entwicklungsländern» zusammen mit dem «Schweizerischen Tropeninstitut» in Ifakara, im Südosten 
Tansanias, gegründete Ausbildungsstätte für medizinisches und landwirtschaftliches Hilfspersonal erwähnt. Neben der Finanzhilfe kommt immer der Persönlichkeit der Experten, die in erster Linie über Sprachkenntnisse und Einfühlungsvermögen verfügen müssen, entscheidendes Gewicht zu. Die Gegenüberstellung von erfolgreichen und mißlungenen Versuchen weckt das kritische Verhalten und die Mitverantwortung im Schüler. Aus sachlich begründeten Urteilen über solche aktuellen Probleme erwächst allmählich die Meinungs- und Gesinnungsbildung. Der Zürcher Hochschulgeograph Prof. Dr. H. Gutersohn formuliert treffend (Lit. 5, S. 404): "Die Entwicklungshilfe ist eine für die ganze Welt bedeutsame Aufgabe, sie ist vielleicht die Aufgabe unserer Zeit. Sie ist vielleicht die letzte große Anstrengung der freien Welt, sich zu behaupten. Mögen wir dieser Aufgabe gewachsen sein!»

\section{Literatur}

1. Basellandschaftliche Schulnachrichten. Hrsg. von der Erziehungsdirektion des Kt. Basellandschaft. 23. Jg., H. 5, April 1962.

2. Bösiger, K.: Basel und seine Region in der Schulgeographie. Regio Basiliensis, H. IV/2, 1965.

3. Bösiger, K.: Die Geographie als Konzentrationsfach in Schulkolonien. Geographica Helvetica, Nr. 3, 1965.

4. Egli, E.: Flugbild Europas. Artemis, Zürich 1958. 5. Entwicklungshilfe. Beiträge zum Gymnasium Helveticum, Bd. 16, H. 5, 1961/62.

6. Guggenbühl, A.: Die Schweizer sind anders. Schweizer Spiegel Verlag, Zürich 1967.

7. Letsch, E.: Geographie an Mittelschulen und nationale Erziehung. Geographischer Anzeiger, Jg. 1916.

8. Schnass-Gerbershagen: Der Erdkundeunterricht. 3. Aufl., Dürrsche Buchhandlung, Bonn 1964.
9. Spranger, E.: Der Bildungswert der Heimatkunde. Reclam, Stuttgart 1958.

\section{Anmerkung}

1 Das am 30. August 1968 im Rahmen des Zyklus «Seminar im Seminar» für die Seminaristen aller Stufen in Basel gehaltene Referat wird hier in etwas gekürzter Fassung einem weiteren Kreise unterbreitet.

\section{Résumé}

Attaché au sol et ouvert au monde - voies et possibilités de la formation et de l'éducation dans l'enseignement de la géographie

"Attaché au sol» et «ouvert au monde» ne signifie pas une opposition, mais une réciprocité. La base de chaque enseignement de la géographie devrait être la connaissance du pays natal, c'est-à-dire de la Suisse. C'est cette connaissance profonde du pays natal qui crée les notions fondamentales pour la géographie en général. - Quelques exemples concernent la protection de la nature et de la civilisation et culture du pays. Par d'autres exemples nous avons essayé d'illustrer des questions méthodologiques: Comment traiter les problèmes d'une agglomération comme celle de la région de Bâle? Comment faire comprendre aux élèves les problèmes d'une commune rurale, dans une colonie scolaire, par exemple? Le travail actif des élèves, soit seuls ou en équipe, est d'une grande importance. Le fait alarmant qu'un certain nombre des jeunes ne cultivent plus nos traditions et notre culture pose des problèmes fort graves. Enfin, la situation de l'Europe entre les puissances mondiales et les régions extraeuropéennes illustre notre dépendance réciproque et la nécessité d'une intégration modérée d'une part, d'une assistance technique bien considérée, mais énergique d'autre part. 